\title{
MOBllity assessment with modern TEChnology in older patients' real-life by the General Practitioner: the MOBITEC-GP study protocol
}

Mareike Münch', Robert Weibel ${ }^{2}$, Alexandros Sofios ${ }^{2}$, Haosheng Huang $^{2}$, Denis Infanger ${ }^{1}$, Erja Portegijs ${ }^{3}$, Eleftheria Giannouli', Jonas Mundwiler ${ }^{4}$, Lindsey Conrow², Taina Rantanen ${ }^{3}$, Arno Schmidt-Trucksäss', Andreas Zeller ${ }^{4}$ and Timo Hinrichs ${ }^{1 *}$ (D)

\begin{abstract}
Background: Mobility limitations in older adults are associated with poor clinical outcomes including higher mortality and disability rates. A decline in mobility (including physical function and life-space) is detectable and should be discovered as early as possible, as it can still be stabilized or even reversed in early stages by targeted interventions. General practitioners (GPs) would be in the ideal position to monitor the mobility of their older patients. However, easy-to-use and valid instruments for GPs to conduct mobility assessment in the real-life practice setting are missing. Modern technologies such as the global positioning system (GPS) and inertial measurement units (IMUs) - nowadays embedded in every smartphone - could facilitate monitoring of different aspects of mobility in the GP's practice.

Methods: This project's aim is to provide GPs with a novel smartphone application that allows them to quantify their older patients' mobility. The project consists of three parts: development of the GPS- and IMU-based application, evaluation of its validity and reliability (Study 1), and evaluation of its applicability and acceptance (Study 2).

In Study 1, participants (target $N=72$, aged $65+, \geq 2$ chronic diseases) will perform a battery of walking tests (varying distances; varying levels of standardization). Besides videotaping and timing (gold standard), a high-end GPS device, a medium-accuracy GPS/IMU logger and three different smartphone models will be used to determine mobility parameters such as gait speed. Furthermore, participants will wear the medium-accuracy GPS/IMU logger and a smartphone for a week to determine their life-space mobility. Participants will be re-assessed after 1 week. In Study 2, participants (target $N=60$, aged $65+, \geq 2$ chronic diseases) will be instructed on how to use the application by themselves. Participants will perform mobility assessments independently at their own homes. Aggregated test results will also be presented to GPs. Acceptance of the application will be assessed among patients and GPs. The application will then be finalized and publicly released.
\end{abstract}

Discussion: If successful, the MOBITEC-GP application will offer health care providers the opportunity to follow their patients' mobility over time and to recognize impending needs (e.g. for targeted exercise) within pre-clinical stages of decline.

Keywords: Aging, General practice, Multimorbidity, Walking speed, Mobility limitation, Smartphone, Geographic information systems, Inertial sensors, Health promotion, Spatial behavior

\footnotetext{
*Correspondence: timo.hinrichs@unibas.ch

1 Department of Sport, Exercise and Health, Division of Sports and Exercise

Medicine, University of Basel, Birsstrasse 320 B, 4052 Basel, Switzerland

Full list of author information is available at the end of the article
}

(c) The Author(s). 2019 Open Access This article is distributed under the terms of the Creative Commons Attribution 4.0 International License (http://creativecommons.org/licenses/by/4.0/), which permits unrestricted use, distribution, and reproduction in any medium, provided you give appropriate credit to the original author(s) and the source, provide a link to the Creative Commons license, and indicate if changes were made. The Creative Commons Public Domain Dedication waiver (http://creativecommons.org/publicdomain/zero/1.0/) applies to the data made available in this article, unless otherwise stated. 


\section{Background}

\section{Multimorbidity and health care for older adults}

Demographic transformation and increasing life expectancy in industrialized countries comes along with a growing number of older adults suffering from chronic health conditions, such as osteoarthritis, coronary heart disease, diabetes mellitus, or dementia [1]. The proportion of patients with "multimorbidity", usually defined as the cooccurrence of at least two chronic conditions [2], grows accordingly. Figures on the prevalence of multimorbidity among over 65-year-olds vary between 40 and 85\% [2-5]. Multimorbidity is strongly associated with poor clinical outcomes, including reduced quality of life [6], increased risk of inappropriate medication with major side-effects $[5,7]$ as well as high mortality and disability rates $[8,9]$. The burden of disease is substantial for the affected patients and their relatives, and also for the healthcare system [10-12].

The simple definition of multimorbidity hardly accounts for the complex relationships between concurrent chronic diseases and the difficulties in managing multimorbid patients. It has been recognized that these patients' needs are insufficiently served by traditional ways of health care provision [12-14]. One reason is the disease-oriented rather than integrated approach towards managing patients with multimorbidity $[15,16]$. Disease-specific guidelines are often contradictory and impractical when applied to multimorbid patients. Disease-oriented management often results in polypharmacy and knowledge of potential side effects, hazards and harm of interventions is insufficient [15]. This lack of knowledge is even more striking, as it is not clear whether traditional improvements in outcomes, for example mortality, are attainable and desirable in patients with multimorbidity [16]. Patient and relative preferences are frequently unmet, as are patient-oriented outcomes such as improving physical functioning and maintaining independence $[17,18]$. There is even evidence that the association between multimorbidity and mortality is lost when adjusted for functional impairment [19]. Hence, there is increasing awareness of the importance of physical functioning as a basic integrator of older adults' health and as a major health outcome; optimizing functional status has been recognized as a central goal for all persons with chronic illness [20].

\section{Mobility}

"Mobility" is a central element of physical functioning [21, 22]. It has been defined comprehensively as "the ability to move oneself (either independently or by using assistive devices or transportation) within environments that expand from one's home to the neighbourhood and to regions beyond" ([23], p. 444). Hence, measures that have been used to characterize a person's mobility include tests of "physical function" [24], and assessments of "life space" [25].
Lower extremity physical function can be measured by simple tests such as stop-watch measured gait speed, balance measures such as maintaining the tandem stand for $10 \mathrm{~s}$, or simple assessments of muscle power such as timing of 5 sit-to-stand cycles [24, 26]. In relation to a person's mobility, gait speed is one of the most central functional parameters. In prospective studies, poor physical function as well as reduced gait speed alone have been shown to be highly predictive of falls [27, 28], dependency in basic activities of daily living [29-31], healthcare utilization [32-34], and mortality [35, 36]. Reduced physical function has been shown to foster social isolation [37], anxiety and depression [38], and to be associated with an overall reduced quality of life [39]. Based on the existing evidence from large-scale prospective studies, clinical cut-offs for functional measures, indicating an increased risk for falls or an increased risk for mobility disability, have been established [40-42], so that these measures have found their way into routine geriatric inpatient care and rehabilitation settings.

Life-space, the "spatial extent in which a person moves within a specified period" ([43], p. 155), encompasses "the interaction between intrinsic capabilities of the person and the demands of the extrinsic environment" ([43], p. 155). It has been shown that restricted life-space is predictive of disability in activities of daily life (ADL) [44], nursing home admission [45] and mortality [46]. Until now, epidemiological studies mostly relied on questionnaires to measure life-space. However, the geospatial information gained from questionnaires is rather crude. As an example, the University of Alabama at Birmingham (UAB) Study of Aging Life-Space Assessment (LSA) assesses the extent of a person's movement (within the past 4 weeks) categorized into 5 spatial levels, ranging from the participant's bedroom to places outside the participants' home town [25]. While the independent predictive value of life-space on different health-related outcomes has been shown repeatedly [45-48], recommendations on clinical cut-offs are currently sparse (e.g .[ 49]) and measures of life-space have not yet found their way into clinical practice.

\section{The necessity to assess mobility}

Measures of functional status have been suggested to inform health care payment systems [50] and they are central components of decision trees advocated by current fall prevention guidelines $[27,41]$. In early stages of decline, physical function can be stabilized or even reversed by targeted intervention such as exercise programmes [51]. Life-space, and thereby participation in social life, can be maintained by adapting the environment to the patients' needs (e.g. by providing assistive devices, adapting patients' homes or providing social support). Similar to chronic illness, physical functioning is dynamic in nature [52]; mobility should 
therefore be routinely monitored to account for the dynamic interactions between physiological systems and the environments of daily life over time. It has even been suggested that functional status should be considered the "sixth vital sign" in addition to the conventional vital signs (body temperature, pulse etc.) [20, 53, 54].

\section{The general practitioner's practice - an opportunity for targeting older adults' mobility}

As mentioned, physical function tests have found their way into routine geriatric inpatient care and rehabilitation settings, but general practitioners (GPs) do not routinely assess physical function as part of their older patients' management $[55,56]$. This deficiency in administering assessments might occur for a number of reasons: physical function assessments (e.g. the measurement of $4 \mathrm{~m}$ gait speed) require standardized equipment, space and training; they are time- and therefore cost-intensive. There is a lack of reimbursement and particularly in patients with multiple chronic conditions, GPs have to weigh the realization of functional assessments against other preventive and therapeutic services $[57,58]$. These considerations are in contrast to the ideal position that GPs would have to monitor their older patients' mobility. GPs are among the few persons who have regular access to community-dwelling and mobility-limited older adults $[59,60]$; they often have established long-lasting, ongoing and trusting relationships with their older patients [61]. GPs are also able to appraise the results of a mobility assessment against the background of their patients' overall health status and medical history.

\section{New opportunities offered by modern technologies - observing mobility in the "real-life" setting}

Modern technologies such as Global Navigation Satellite Systems (GNSS) - including various satellite systems such as GPS, GLONASS, Galileo and Beidou - and inertial measurement units (IMUs) - including accelerometer, gyroscope and magnetometer - offer the chance to implement measures of mobility (physical function and life-space) into routine primary health care and to follow-up on older patients' mobility over time. In contrast to traditional assessment tools, these technologies allow for observation of older adults function in real-life settings and in interaction with their environment $[62,63]$. It has been criticized in the past that laboratory-based functional assessments only have limited value in predicting older adults' real-life mobility behaviour [64]. GNSS technology and IMUs are nowadays embedded in every modern smartphone, a device that has become popular and shows much promise in improving the health care of older adults. High availability, objectivity and low additional costs might facilitate widespread future use.

While high-end, handheld GPS devices can achieve decimetre accuracy, typical consumer GPS devices or smartphones will achieve best-case accuracies of 2-3 m under normal conditions [65]. Latest smartphone models are also meant to achieve decimeter-level positioning accuracy in post-processing mode, due to the ability to use dual frequency GPS/GALILEO [66]. That is, consumerlevel products are starting to reach a positioning accuracy level where accurate and robust movement parameter derivation (e.g. distance, speed, acceleration etc.) is becoming feasible, particularly for distances that exceed the GPS error by at least one order of magnitude, that is, several dozens to some hundred meters. So far, however, the use of GPS-derived movement parameters to quantify physical performance has mainly been limited to team sports $[67,68]$. In the health sciences, most applications have been confined to using GPS fixes to estimate individuals' life-spaces [69], often linking these to active transport and body weight [70]. There are few reports on applications of GPS-derived movement parameters in patient populations. A limited number of studies have shown the reliability and applicability of GPS-derived measures in a sample of patients with peripheral arterial disease [71, 72].

Locomotion speed can already be accurately extracted from IMU readings over shorter distances [73, 74]. Since GPS and IMUs are standard components of contemporary smartphones, there is potential for smartphone-based measurements to replace or complement traditional usually stopwatch timed - walking tests over short distances (e.g. $10 \mathrm{~m}$ ). In contrast to GPS devices, IMUs do not require satellites visibility, i.e. they can be used both indoors and outdoors. On the other hand, GPS is the optimal method to determine locations over longer periods of time, and therefore the optimal method to assess lifespace mobility.

\section{Steps to be taken}

Though the sources quoted above have demonstrated the potential of GPS- and IMU-derived measures, they still have to be adapted to the new context and tested for their reliability, validity, applicability and acceptance before they can be routinely used by GPs to monitor their patients' mobility (physical function and life-space). There is also a need to evaluate how GPS- and IMUderived mobility measures relate to traditional physical function tests.

\section{Methods and design}

\section{Aims and objectives}

The overall goal of the MOBITEC-GP project is to provide GPs with the possibility to assess their older 
patients' mobility using a collection of different mobility parameters (related to physical function and life space).

The aims of the project are:

1. To develop an easy-to-use GPS- and IMU-based smartphone application that allows GPs to quantify and appraise their older patients' mobility ("Development");

2. To evaluate the application's validity and reliability ("Study 1");

3. To evaluate the new tools' applicability and acceptance among GPs and patients ("Study 2").

An iterative development and evaluation process is required to fulfil all aims; it is illustrated in Fig. 1. The development phases include requirement analyses among GPs, patients and experts. While the first development phase focuses on the app's measurement properties, the second development phase focusses on user interface design. The app will be specifically designed to create a patient-GP partnership, i.e. the patient collects data of daily functioning and the GP interprets and explains the data to the patient, and plans the necessary measures (such as referral to a comprehensive fall risk assessment or to an exercise programme, home adaptations or provision with adaptive devices) together with the patient.

Specific objectives of Study 1 are:

1) To assess the test-retest reliability of GPS/IMU-derived physical function measures (smartphone, medium-accuracy GPS/IMU device, high-end GPS device; see section 'Measurements in Study 1' below) obtained during standardized short-distance mobility tasks (10 m and $50 \mathrm{~m}$ walks; "short distance controlled condition") and their validity against "gold standard" (timing, video-recording).

2) To assess the test-retest reliability of GPS/IMU-derived physical function measures (smartphone, medium-accuracy GPS/IMU device, high-end GPS device) obtained during a standardized longdistance mobility task (400 m walk on a given track; "long-distance controlled condition") and their validity against "gold standard" (timing, videorecording).

3) To assess the test-retest reliability of GPS/IMU-derived physical function measures (smartphone, medium-accuracy GPS/IMU device, high-end GPS device) obtained during a semi-standardized mobility task (30-min stroll in the park; "semi-controlled

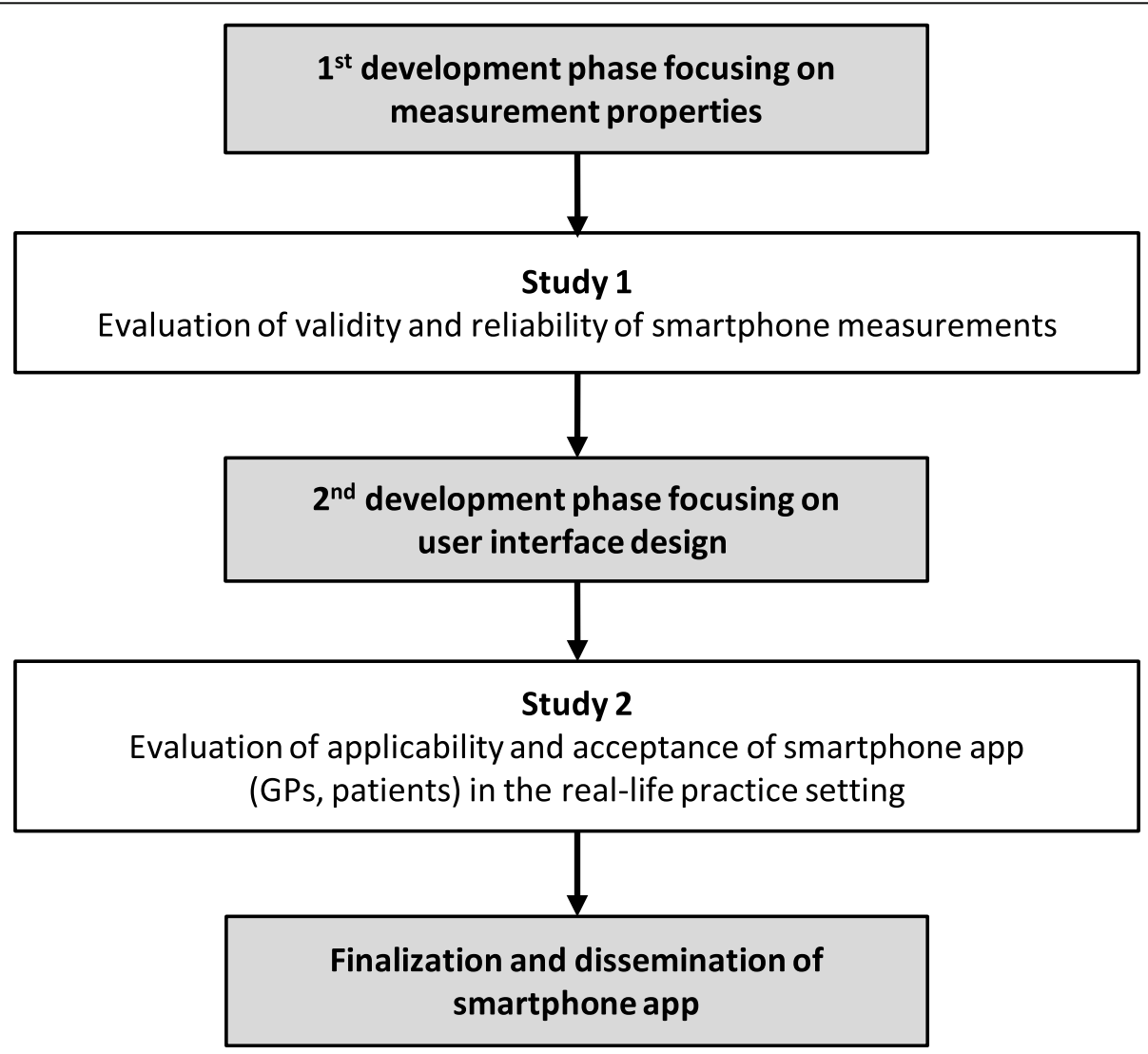

Fig. 1 Iterative process of development and evaluation of the new smartphone application 
condition") and their concurrent validity (against traditional physical function tests).

4) To assess the test-retest reliability of GPS/IMU-derived life-space measures (smartphone, mediumaccuracy GPS/IMU-device) during a one-week measurement period around participants' homes (uncontrolled "real-life" condition) and their concurrent validity (against traditional physical function tests).

5) To assess whether measurement properties (validity, reliability) vary by key participant characteristics (including demographic characteristics, anthropometrics, and physical function measures).

6) To assess whether measurement results (physical function and life-space) vary by key participant characteristics (including demographic characteristics, physical activity level, gait efficacy, fall status, and morbidity) to generate hypotheses for subsequent large-scale epidemiological studies.

7) To assess the inter-instrument agreement between a high-end GPS device, a medium-accuracy GPS/ IMU device and three different smartphone models, including agreement between the different smartphone models and agreement between smartphone wearing locations.

8) To define cut-offs for indicators of different mobility levels against established clinical cut-offs based on traditional physical function measures (from existing epidemiological studies and current fallprevention guidelines).

Specific objectives of Study 2 are:

9) To assess the clinical applicability of unsupervised GPS/IMU measurements with the smartphone (fitted with the new application) in the real-life general practice setting.

10) To assess usability and acceptance by GPs and patients.

11) To assess participant characteristics that are associated with the proportion of clinically usable unsupervised GPS/IMU measurements.

\section{Design}

Study 1 and Study 2 will both be observational. Study 1 will be a validity and reliability study. At baseline $\left(\mathrm{T}_{0}\right)$, a battery of tests (visit at study center) as well as a 1-week measurement around participants' homes will be performed. Cross-sectional analyses of baseline values will be used to evaluate validity of the GPS and IMU-based measurements as well as the inter-instrument agreement between devices of different accuracy levels as well as between different smartphone models. Participants will be assessed again 1 week after the initial assessment $\left(T_{1}\right)$ to evaluate test-retest-reliability of the GPS/IMU measurements. $T_{1}$ includes a visit at the study center and a 1-week measurement around participants' homes.

Study 2 will be an applicability study: Patients equipped with a smartphone (fitted with the new application) will independently perform mobility measurements around their homes for 1 week. The proportion of technically satisfactory GPS/IMU recordings will be determined. Reasons for unsuccessful recordings will be assessed and analysed. Usability and acceptance by GPs and patients will be evaluated.

\section{Inclusion criteria}

Both studies target community-dwelling older patients from general practice with multiple chronic conditions, aged 65 or above. The target number of participants for Study 1 is 72 , the target number of participants for Study 2 is 60. Participants have to be diagnosed (self-report) with at least two of the following chronic diseases (according to the "Self-Administered Comorbidity Questionnaire" (SCQ) [75, 76]): heart disease, high blood pressure, lung disease, diabetes, ulcer or stomach disease, kidney disease, anemia or other blood disease, cancer, depression, osteoarthritis, degenerative arthritis, back pain and/or rheumatoid arthritis. Participants have to be able to perform a 30-min outdoor walk at their own pace, with or without breaks, with or without walking aid, but without the help of another person (self-report). Persons who are incapable of judgment and persons who are unable to follow procedures or have insufficient knowledge of the German language will be excluded. Participants will have to provide written informed consent.

\section{Recruitment}

The recruitment strategy will be twofold. On one hand, participants will be recruited through GP practices. Patients who attend their GP within a given timeframe, are capable of judgement and aged 65 or above will be asked by the GP for their general interest in participating in a study on "mobility". If they agree they will be informed about the study and assessed for eligibility by a research team member. All eligible patients will be invited to participate. On the other hand, participants will be recruited through presentations about the project in local senior citizen gatherings, individual invitations to persons who expressed interest in participating in studies from our institute, as well as handing out information brochures about the study and individually approaching older adults in settings such as pharmacies, churches, and senior sports groups.

GP practices will be recruited from a network of practices associated with the Center of Primary Health Care, University of Basel. 


\section{Ethical considerations and ethical approval}

The performed measurements will only include everyday tasks (such as walking at preferred, habitual pace or rising from a chair), so that they do not involve an increased cardiovascular or musculoskeletal risk compared to everyday activity. During the walking tests, participants will be allowed to take a break at any time.

The new smartphone application will be designed in a way that GPs are only provided with summary measures of physical function and life-space mobility. GPs will not have an insight into the raw movement data or locations that older adults have visited.

The research is carried out in compliance with the Helsinki Declaration. Data generation, transmission, storage and analysis of health related personal data will follow the current Swiss legal requirements for data protection. The project was approved by the Ethics Committee of Northwestern and Central Switzerland (EKNZ) (Reg.-No. 2018-02257).

\section{Measurements in study 1 GPS and IMU-based measures}

GPS and IMU-based measurements will take place at two points in time $\left(\mathrm{T}_{0}\right.$ and $\left.\mathrm{T}_{1}\right)$ at the study center. Four different mobility tasks including GPS and IMU-based measurements will be performed. Tasks a) to c) will take place at the study center; Task d) will take place in the week immediately after each visit around participants' homes. The devices used in Study 1 will include a highend GPS device (Trimble GeoExplorer $5 \mathrm{~T}$, Trimble Inc., Sunnyvale, CA, USA); a medium accuracy GPS/IMU device (uTrail, CDD Ltd., Athens, Greece) and three different models of contemporary dual-antenna smartphones (Samsung Galaxy S8, Xiaomi Mi 8, Apple iPhone SE).

Task a): two supervised walks of $10 \mathrm{~m}$ and $50 \mathrm{~m}$ length, at self-selected, habitual pace on an outdoors athletics track (short-distance controlled condition). These tasks will be videotaped (Garmin VIRB XE, Garmin Ltd., Olathe, KS, USA) and timed (light barrier system; BROWER Timing Systems, Draper, UT, USA). Participants will be equipped with the high-end GPS device, the medium accuracy GPS/IMU device and the three smartphones. The three different smartphone models will be worn at three different locations (waist belt, sling bag, and neck pouch). This will be used to assess the influence of wearing location and model on the results.

Task b): a supervised $400 \mathrm{~m}$ walk at self-selected, habitual pace on an outdoors athletics track (long-distance controlled condition). This task will also be videotaped and timed. Again, participants will be equipped with the high-end GPS device, the medium-accuracy GPS/IMU device and three smartphones.

Task c): an unsupervised 30-min stroll in a park at self-selected, habitual pace without a given track (semi- controlled condition). Participants will be equipped with the high-end GPS device, the medium-accuracy GPS/ IMU device and three smartphones.

Task d): a one-week measurement period around participants' homes without any specified tasks (uncontrolled "real-life" condition). Participants will be equipped with an uTrail device, a smartphone (random choice of 1 of the 3 models) and a wrist-worn activity tracker (see section 'Traditional physical function tests and physical activity' below).

During all tasks, participants will be allowed to stop and rest any time. There will be no recommendation about minimum or maximum duration of stops. Weather conditions (temperature and cloud cover) will be documented at $\mathrm{T}_{0}$ and $\mathrm{T}_{1}$; these parameters will be considered for sensitivity analyses as they might affect reliability.

Data processing will include:

Tasks a) and b): Gait speed (average, average per section, and maximum), number of steps, number and duration of stops, and greatest distance between stops will be derived from the raw GPS/IMU data. Average gait speed (distance/time) and number of steps will also be derived from timing and video recording.

Task c): Gait speed (average, average per section, and maximum), number of steps, number and duration of stops, and greatest distance between stops will be derived from the raw GPS/IMU data.

Task d): Previously suggested approaches to derive summary measures of life-space from GPS data will be used (including convex hull and standard deviation ellipse) [77].

\section{Traditional physical function tests and physical activity}

In addition to the GPS/IMU-based measurements, physical function will also be assessed by a battery of traditional functional geriatric tests at $\mathrm{T}_{0}$ (before the GPS/ IMU measurements) at the study center: short physical performance battery [24], single-leg stance [78], timed "Up \& Go" [26], and grip strength (Jamar plus dynamometer, Sammons Preston, Bolingbrook, IL) [79]. Additionally, habitual physical activity will be assessed by the use of a wrist-worn activity tracker (vivofit 2, Garmin Ltd., Olathe, KS, USA) the week after $\mathrm{T}_{0}$.

\section{Further measures}

The following participant characteristics will be assessed within the baseline $\left(\mathrm{T}_{0}\right)$ assessment (self-report): sex, age, residential area (urban, suburban or rural), living condition (alone or with someone else), socio-economic status (financial hardship and years of education), current walking ability (no walking aid, cane or rollator), frequency of falls (12-month recall) [80], sports participation [81], gait efficacy (modified Gait Efficacy Scale) 
[82, 83], perceived health status and disability (WHODAS 2.0 12-item version) [84], and chronic diseases (according to the inclusion criteria, see above). Height and weight will be measured by a trained assessor.

Participants will be given a diary for the one-week measurement, where they will note how often they left their homes, if they carried the GPS device and smartphone and if they have worn the activity tracker for at least $10 \mathrm{~h}$ a day.

\section{Measurements in study 2 Smartphone measurements}

Study 2 will use smartphones fitted with the newly developed application. Patients will be instructed by a research team member on how to use the application. The application will be mostly self-explaining. Participants will be asked to take along the smartphone fitted with the application for a 1-week period. They should independently perform one continuous GPS/IMU measurement during a 30-min stroll in a park of their choice at self-selected, usual pace (to determine gait speed). Furthermore, participants' position will be recorded by GPS over this period to determine life-space.

\section{Further measures}

Sex, age, height and weight, residential area (urban, suburban or rural; self-reported) and current walking ability (self-reported) will be documented. After finishing the 1 week measurements, participants will be contacted by a research member and they will be asked to rate the usability of the tool.

Furthermore, aggregated results will be presented to participating GPs. They will be asked to rate the usability of the tool and the usefulness of the obtained information.

\section{Statistical analyses}

Participant characteristics (demographics, history of falls, chronic conditions etc.) will be analysed descriptively.

\section{Study 1}

Objectives 1 and 2 Test-retest reliability and validity will be assessed by calculating Intraclass Correlation $\mathrm{Co}$ efficients (ICCs) [85]. Agreement will also be assessed by performing Bland-Altman-Analyses [86]. In addition to ICCs, recently developed information-based measures to quantify reliability will be considered [87].

Objectives 3 and 4 Test-retest reliability will be assessed by calculating ICCs [85] (as well as information-based measures [87]). Again, Bland-Altman-Analyses will be performed [86]. Concurrent validity will be assessed by estimating correlations or associations (depending on the level of measurement of the respective parameters).

Objective 5 The above mentioned estimation procedures will be performed stratified by subgroups (based on demographic characteristics, anthropometrics, and physical function measures).

Objective 6 Measures of physical function and lifespace will be analysed (descriptive statistics and 95\% confidence intervals) for the total sample and stratified by subgroups (based on demographic characteristics, physical activity level, gait efficacy, and morbidity).

Objective 7 Inter-instrument agreement (as well as agreement between wearing locations) will be assessed by calculating ICCs [85] (as well as information-based measures [87]) and by performing Bland-AltmanAnalyses [86].

Objective 8 Inter-rater agreement between levels of mobility based on traditional measures and the new smartphone-based measures will be assessed using Cohen's Kappa [88]. Optimal cut-offs for the new measures will be chosen such that Cohen's Kappa is maximized.

\section{Study 2}

Objective 9 The proportion (with 95\% confidence interval) of satisfactory recordings (according to predefined criteria) [71] for the estimation of both life-space (continuous 1-week measurement) and physical function (30-min stroll in the park) will be calculated and presented for the total sample as well as stratified by relevant subgroups (defined based on findings of Study 1). Reasons for unsatisfactory recordings will be analysed. Furthermore, the obtained physical function and lifespace data will be analysed descriptively for the total sample and stratified by relevant subgroups.

Objective 10 Acceptance by GPs and patients will be analysed by descriptive statistics.

Objective 11 Logistic regression models will be used to assess participant characteristics that are associated with the proportion of satisfactory recordings.

\section{Sample size calculation \\ Study 1}

The primary analysis is the estimation of the ICC (95\% confidence interval) between the $T_{0}$ and $T_{1}$ value of the GPS/IMU-derived average walking speed during the 30min stroll in the park. To estimate an ICC of 0.9 with an 
expected 95\% confidence interval width of $0.1,61$ patients will be necessary (underlying assumption based on results of Gernigon et al. [72]) [89]. Accounting for an anticipated drop-out rate of $15 \%$, the target sample size is 72 .

\section{Study 2}

The primary analysis is the estimation of the proportion of satisfactory GPS recordings from the 30-min stroll in the park. Assuming that $80 \%$ of GPS measurements will be successful (based on results of Gernigon et al. [71] (85\%), but being more conservative due to the higher age and morbidity of participants in our study), 60 patients are needed to achieve an expected width for a $95 \%$ confidence interval of 0.2 . We will use the Wilson interval which is an improvement over the traditional Wald-type interval [90]. No drop-outs are assumed for this study because there is no follow-up.

\section{Discussion}

MOBITEC-GP will provide the prerequisites for a novel tool for primary health care, offering GPs the opportunity to routinely assess their older adult patients' mobility and to recognize impending needs within pre-clinical stages of decline.

Making use of technologies that are embedded in smartphones offers a cost-effective opportunity as these devices are already available and no "extra device" is needed. The proportion of smartphone owners in the population is growing rapidly: between 2013 and 2018 it has increased from 70 to $90 \%$ in the general Swiss population [91]. Even though an US survey (2015) has shown that the proportion of smartphone users among those aged $65+(27 \%)$ is still markedly lower than in the younger age categories (54\% in those aged 50 to 64 ) [92], it can be expected that smartphone use in old age will increase in the future - substantially but not exclusively due to the aging of middle aged smartphone users [92]. A recent survey among patients after surgery showed that even though older patients were less likely to have a smartphone, they were just as interested and willing as their younger counterparts to engage with mobile health technologies [93].

Furthermore, the measurement as designed in MOBITEC-GP do not need supervision and thus are not personnel consuming. This will facilitate the use of these measures in primary health care as well as in future large-scale cohort studies, aiming to determine or confirm clinical cut-offs for targeted intervention. The advent of electronic medical records will provide an opportunity to link in situ mobility monitoring with medical records and to integrate algorithms that automatically detect critical values for fall risk and social isolation. The new tool could also provide an objective measure for other health care professionals, such as social workers, occupational therapists or physiotherapists, when life-changing decisions such as moving into a nursing home have to be made; or alternatively, ambulatory services have to be planned in order to enable older adults to stay in their own homes. Finally, the new tool might facilitate further research on the relationship between older adults' functioning and their (geographical and social) environment.

\section{Abbreviations \\ GNSS: Global Navigation Satellite Systems; GP: General Practitioner; GPS: Global Positioning System; ICC: Intraclass Correlation Coefficient: IMU: Inertial Measurement Unit \\ Acknowledgements \\ Not applicable. \\ Authors' contributions \\ Conceptualisation of the project and funding acquisition: TH (Principal Investigator), RW, HH, AZ, DI, AS-T, EP and TR. Contribution to study design: all authors. Implementation of study, quality assurance and supervision: $\mathrm{TH}$ and RW. Project management: TH, MM and EG. Recruitment and support of GPs: AZ and JM. Recruitment of participants: MM and EG. Data collection and data management: $M M$ and $E G$. Technical design of smartphone app and data processing steps: RW, HH, AS and LC. Programming of smartphone app: AS. Processing of GPS and IMU data: AS and LC. Statistical analysis plan: DI and TH. Manuscript draft: TH, RW, HH, DI and MM. Critical revision and final approval of manuscript: all authors.}

\section{Funding}

This project is financed by the Velux Stiftung (TH; Project No. 1155). Funders have no role in the study design and execution of the study, analysis, and interpretation of data and in writing the manuscript.

\section{Availability of data and materials}

After completion of the project, data will be available from the corresponding author on reasonable request. The source code of the app as well as the analysis software will be publicly available as 'open source'.

\section{Ethics approval and consent to participate}

MOBITEC-GP follows the principles of the Declaration of Helsinki. The Ethics Committee of Northwestern and Central Switzerland (EKNZ) approved the project (Reg.-No. 2018-02257). All participants have to provide written informed consent.

\section{Consent for publication}

Not applicable.

\section{Competing interests}

The authors declare that they have no competing interests.

\section{Author details}

${ }^{1}$ Department of Sport, Exercise and Health, Division of Sports and Exercise Medicine, University of Basel, Birsstrasse 320 B, 4052 Basel, Switzerland.

${ }^{2}$ Geographical Information Systems Unit, Department of Geography, University of Zürich, Zürich, Switzerland. ${ }^{3}$ Gerontology Research Center and Faculty of Health Sciences, University of Jyväskylä, Jyväskylä, Finland. ${ }^{4}$ Center for Primary Health Care, University of Basel, Basel, Switzerland.

Received: 5 December 2019 Accepted: 11 December 2019

Published online: 19 December 2019

\section{References}

1. Marengoni A, Angleman S, Melis R, Mangialasche F, Karp A, Garmen A, Meinow B, Fratiglioni L. Aging with multimorbidity: a systematic review of the literature. Ageing Res Rev. 2011;10(4):430-9.

2. van den Akker M, Buntinx F, Metsemakers JFM, Roos S, Knottnerus JA. Multimorbidity in general practice: prevalence, incidence, and determinants 
of co-occurring chronic and recurrent diseases. J Clin Epidemiol. 1998;51(5): $367-75$.

3. Fortin M, Bravo G, Hudon C, Vanasse A, Lapointe L. Prevalence of multimorbidity among adults seen in family practice. Ann Fam Med. 2005; 3(3):223-8.

4. Weiss CO, Boyd CM, Yu QL. Patterns of prevalent major chronic disease among older adults in the United States. JAMA. 2007;298(10):1160-2.

5. Kostev K, Jacob L. Multimorbidity and polypharmacy among elderly people followed in general practices in Germany. Eur J Intern Med. 2018;55:66-8.

6. Fortin M, Bravo G, Hudon C, Lapointe L, Dubois MF, Almirall J. Psychological distress and multimorbidity in primary care. Ann Fam Med. 2006;4(5):417-22.

7. Zhen CL, Sangl J, Bierman AS, Miller MR, Friedman B, Wickizer SW, Meyer GS. Potentially inappropriate medication use in the community-dwelling elderly - findings from the 1996 medical expenditure panel survey. JAMA. 2001;286(22):2823-9.

8. Gijsen R, Hoeymans N, Schellevis FG, Ruwaard D, Satariano WA, van den Bos GAM. Causes and consequences of comorbidity: a review. J Clin Epidemiol. 2001;54(7):661-74.

9. Pompei P, Charlson ME, Douglas RG. Clinical assessments as predictors of one year survival after hospitalization - implications for prognostic stratification. J Clin Epidemiol. 1988;41(3):275-84.

10. Rochon PA, Katz JN, Morrow LA, McGlincheyBerroth R, Ahlquist MM, Sarkarati M, Minaker KL. Comorbid illness is associated with survival and length of hospital stay in patients with chronic disability - a prospective comparison of three comorbidity indices. Med Care. 1996;34(11):1093-101.

11. Flocke SA, Frank SH, Wenger DA. Addressing multiple problems in the family practice office visit. J Fam Pract. 2001;50(3):211-6.

12. Epping-Jordan JE, Pruitt SD, Bengoa R, Wagner EH. Improving the quality of health care for chronic conditions. Qual Saf Health Care. 2004;13(4):299-305.

13. Bodenheimer T, Wagner EH, Grumbach K. Improving primary care for patients with chronic illness. JAMA. 2002;288(14):1775-9.

14. Wagner EH, Bennett SM, Austin BT, Greene SM, Schaefer JK, Vonkorff M. Finding common ground: patient-centeredness and evidence-based chronic illness care. J Altern Complement Med. 2005;11(Suppl 1):S7-15.

15. Tinetti ME, Bogardus ST Jr, Agostini JV. Potential pitfalls of diseasespecific guidelines for patients with multiple conditions. N Engl J Med. 2004;351(27):2870-4.

16. Tinetti ME, Fried T. The end of the disease era. Am J Med. 2004;1 16(3): 179-85.

17. Boyd CM, Darer J, Boult C, Fried LP, Boult L, Wu AW. Clinical practice guidelines and quality of care for older patients with multiple comorbid diseases: implications for pay for performance. JAMA. 2005;294(6):716-24.

18. Fried TR, Tinetti ME, lannone L, O'Leary JR, Towle V, Van Ness PH. Health outcome prioritization as a tool for decision making among older persons with multiple chronic conditions. Arch Intern Med. 2011;171(20):1854-6.

19. St John PD, Tyas SL, Menec V, Tate R. Multimorbidity, disability, and mortality in community-dwelling older adults. Can Fam Physician. 2014; 60(5):e272-80.

20. Bierman AS. Functional status: the six vital sign. J Gen Intern Med. 2001; 16(11):785-6.

21. Satariano WA, Guralnik JM, Jackson RJ, Marottoli RA, Phelan EA, Prohaska TR. Mobility and aging: new directions for public health action. Am J Public Health. 2012;102(8):1508-15.

22. World Health Organisation. International classification of functioning, disability and health: ICF. Geneva: WHO; 2001.

23. Webber SC, Porter MM, Menec VH. Mobility in older adults: a comprehensive framework. Gerontologist. 2010;50(4):443-50.

24. Guralnik JM, Simonsick EM, Ferrucci L, Glynn RJ, Berkman LF, Blazer DG, Scherr PA, Wallace RB. A short physical performance battery assessing lower-extremity function - association with self-reported disability and prediction of mortality and nursing-home admission. J Gerontol. 1994; 49(2):M85-94

25. Baker PS, Bodner EV, Allman RM. Measuring life-space mobility in community-dwelling older adults. J Am Geriatr Soc. 2003;51(11):1610-4.

26. Podsiadlo D, Richardson S. The timed "up \& go" - a test of basic functional mobility for frail elderly persons. J Am Geriatr Soc. 1991;39(2):142-8.

27. Moyer VA, Force USPST. Prevention of falls in community-dwelling older adults: U.S. Preventive Services Task Force recommendation statement. Ann Intern Med. 2012;157(3):197-204.

28. Montero-Odasso M, Schapira M, Soriano ER, Varela M, Kaplan R, Camera LA, Mayorga LM. Gait velocity as a single predictor of adverse events in healthy seniors aged 75 years and older. J Gerontol A Biol Sci Med Sci. 2005;60(10): 1304-9.

29. Lawrence RH, Jette AM. Disentangling the disablement process. J Gerontol B Psychol Sci Soc Sci. 1996;51(4):S173-82

30. Guralnik JM, Ferrucci L, Simonsick EM, Salive ME, Wallace RB. Lowerextremity function in persons over the age of 70 years as a predictor of subsequent disability. N Engl J Med. 1995;332(9):556-61.

31. Rosano C, Newman AB, Katz R, Hirsch CH, Kuller LH. Association between lower digit symbol substitution test score and slower gait and greater risk of mortality and of developing incident disability in well-functioning older adults. J Am Geriatr Soc. 2008;56(9):1618-25.

32. von Bonsdorff M, Rantanen T, Laukkanen P, Suutama T, Heikkinen E. Mobility limitations and cognitive deficits as predictors of institutionalization among community-dwelling older people. Gerontology. 2006;52(6):359-65.

33. Reuben DB, Seeman TE, Keeler E, Hayes RP, Bowman L, Sewall A, Hirsch SH, Wallace RB, Guralnik JM. The effect of self-reported and performance-based functional impairment on future hospital costs of community-dwelling older persons. Gerontologist. 2004;44(3):401-7.

34. Cesari M, Kritchevsky SB, Penninx BW, Nicklas BJ, Simonsick EM, Newman $A B$, Tylavsky FA, Brach JS, Satterfield S, Bauer DC, et al. Prognostic value of usual gait speed in well-functioning older people--results from the health, aging and body composition study. J Am Geriatr Soc. 2005;53(10):1675-80.

35. Bernard SL, Kincade JE, Konrad TR, Arcury TA, Rabiner DJ, Woomert A, DeFriese GH, Ory MG. Predicting mortality from community surveys of older adults: the importance of self-rated functional ability. J Gerontol B Psychol Sci Soc Sci. 1997;52(3):S155-63.

36. Studenski S, Perera S, Patel K, Rosano C, Faulkner K, Inzitari M, Brach J, Chandler J, Cawthon P, Connor EB, et al. Gait speed and survival in older adults. JAMA. 2011;305(1):50-8.

37. Peel C, Baker PS, Roth DL, Brown CJ, Bodner EV, Allman RM. Assessing mobility in older adults: the UAB study of aging life-space assessment. Phys Ther. 2005;85(10):1008-19.

38. Vink D, Aartsen MJ, Schoevers RA. Risk factors for anxiety and depression in the elderly: a review. J Affect Disord. 2008;106(1-2):29-44.

39. Netuveli G, Wiggins RD, Hildon Z, Montgomery SM, Blane D. Quality of life at older ages: evidence from the English longitudinal study of aging (wave 1). J Epidemiol Community Health. 2006;60(4):357-63.

40. Vasunilashorn S, Coppin AK, Patel KV, Lauretani F, Ferrucci L, Bandinelli S, Guralnik JM. Use of the short physical performance battery score to predict loss of ability to walk 400 meters: analysis from the InCHIANTI study. J Gerontol A Biol Sci Med Sci. 2009;64(2):223-9.

41. Panel on Prevention of Falls in Older Persons AGS, British Geriatrics S. Summary of the Updated American Geriatrics Society/British Geriatrics Society clinical practice guideline for prevention of falls in older persons. J Am Geriatr Soc. 2011;59(1):148-57.

42. Shumway-Cook A, Brauer S, Woollacott M. Predicting the probability for falls in community-dwelling older adults using the timed up \& go test. Phys Ther. 2000;80(9):896-903.

43. Tung JY, Rose RV, Gammada E, Lam I, Roy EA, Black SE, Poupart P. Measuring life space in older adults with mild-to-moderate Alzheimer's disease using mobile phone GPS. Gerontology. 2014;60(2):154-62.

44. Portegijs E, Rantakokko M, Viljanen A, Sipilä S, Rantanen T. Identification of older people at risk of ADL disability using the life-space assessment: a longitudinal cohort study. J Am Med Dir Assoc. 2016;17(5):410-4.

45. Sheppard KD, Sawyer P, Ritchie CS, Allman RM, Brown CJ. Life-space mobility predicts nursing home admission over 6 years. J Aging Health. 2013;25(6):907-20

46. Boyle PA, Buchman AS, Barnes LL, James BD, Bennett DA. Association between life space and risk of mortality in advanced age. J Am Geriatr Soc. 2010:58(10):1925-30.

47. Xue QL, Fried LP, Glass TA, Laffan A, Chaves PH. Life-space constriction, development of frailty, and the competing risk of mortality: the Women's health and aging study I. Am J Epidemiol. 2008;167(2):240-8.

48. Lo AX, Brown CJ, Sawyer P, Kennedy RE, Allman RM. Life-space mobility declines associated with incident falls and fractures. J Am Geriatr Soc. 2014; 62(5):919-23.

49. Ullrich P, Werner C, Eckert T, Bongartz M, Kiss R, Feisst M, Delbaere K, Bauer JM, Hauer K. Cut-off for the life-space assessment in persons with cognitive impairment. Aging Clin Exp Res. 2019;31(9):1331-5.

50. Clauser SB, Bierman AS. Significance of functional status data for payment and quality. Health Care Financ Rev. 2003;24(3):1-12. 
51. Pahor M, Guralnik JM, Ambrosius WT, Blair S, Bonds DE, Church TS, Espeland MA, Fielding RA, Gill TM, Groessl EJ, et al. Effect of structured physical activity on prevention of major mobility disability in older adults: the LIFE study randomized clinical trial. JAMA. 2014;311(23):2387-96.

52. Botoseneanu A, Allore HG, Gahbauer EA, Gill TM. Long-term trajectories of lower extremity function in older adults: estimating gender differences while accounting for potential mortality bias. J Gerontol A Biol Sci Med Sci. 2013;68(7):861-8.

53. Cesari M. Role of gait speed in the assessment of older patients. JAMA. 2011:305(1):93-4.

54. Studenski S, Perera S, Wallace D, Chandler JM, Duncan PW, Rooney E, Fox M, Guralnik JM. Physical performance measures in the clinical setting. J Am Geriatr Soc. 2003:51(3):314-22.

55. Calkins DR, Rubenstein LV, Cleary PD, Davies AR, Jette AM, Fink A, Kosecoff J, Young RT, Brook RH, Delbanco TL. Failure of physicians to recognize functional disability in ambulatory patients. Ann Intern Med. 1991;114(6):451-4.

56. Calkins DR, Rubenstein LV, Cleary PD, Davies AR, Jette AM, Fink A, Kosecoff J, Young RT, Brook RH, Delbanco TL. Functional disability screening of ambulatory patients: a randomized controlled trial in a hospital-based group practice. J Gen Intern Med. 1994;9(10):590-2.

57. Tinetti ME, Brach JS. Translating the fall prevention recommendations into a covered service: can it be done, and who should do it? Ann Intern Med. 2012;157(3):213-4.

58. Hinrichs T, Brach M. The general practitioner's role in promoting physical activity to older adults: a review based on program theory. Curr Aging Sci. 2011;5(1):41-50.

59. Saß AC, Wurm S, Ziese T. Inanspruchnahmeverhalten. In: Böhm K, TeschRömer C, Ziese T, editors. Beiträge zur Gesundheitsberichterstattung des Bundes: Gesundheit und Krankheit im Alter. Berlin: Robert Koch-Institut; 2009. p. 134-59.

60. Brach M, Moschny A, Bücker B, Klaassen-Mielke R, Trampisch M, Wilm S, Platen $P$, Hinrichs T. Recruiting hard-to-reach subjects for exercise interventions: a multi-Centre and multi-stage approach targeting general practitioners and their community-dwelling and mobility-limited patients. Int J Environ Res Public Health. 2013;10(12):6611-29.

61. Falvo D, Woehlke P, Deichmann J. Relationship of physician behaviour to patient compliance. Patient Couns Health Educ. 1980;2(4):185-8.

62. Harada K, Lee S, Lee S, Bae S, Harada K, Suzuki T, Shimada H. Objectivelymeasured outdoor time and physical and psychological function among older adults. Geriatr Gerontol Int. 2017;17(10):1455-62.

63. Hirsch JA, Winters M, Ashe MC, Clarke P, McKay H. Destinations that older adults experience within their GPS activity spaces relation to objectively measured physical activity. Environ Behav. 2016;48(1):55-77.

64. Giannouli E, Bock O, Mellone S, Zijlstra W. Mobility in old age: capacity is not performance. Biomed Res Int. 2016;2016:3261567.

65. Pesyna KM, Robert WH, Humphreys WH. Accuracy in the palm of your hand. GPS World. 2015:26(2):16-31.

66. Elmezayen A, El-Rabbany A. Precise point positioning using world's first dual-frequency GPS/GALILEO smartphone. Sensors (Basel). 2019;19(11):2593.

67. Scott MT, Scott TJ, Kelly VG. The validity and reliability of global positioning systems in team sport: a brief review. J Strength Cond Res. 2016;30(5):1470-90.

68. Rampinini E, Alberti G, Fiorenza M, Riggio M, Sassi R, Borges TO, Coutts AJ. Accuracy of GPS devices for measuring high-intensity running in field-based team sports. Int J Sports Med. 2015;36(1):49-53.

69. Hirsch JA, Winters M, Clarke P, McKay H. Generating GPS activity spaces that shed light upon the mobility habits of older adults: a descriptive analysis. Int J Health Geogr. 2014;13:51.

70. Wanner M, Gotschi T, Martin-Diener E, Kahlmeier S, Martin BW. Active transport, physical activity, and body weight in adults: a systematic review. Am J Prev Med. 2012;42(5):493-502.

71. Gernigon M, Le Faucheur A, Noury-Desvaux B, Mahe G, Abraham P, Post GPSSCG. Applicability of global positioning system for the assessment of walking ability in patients with arterial claudication. J Vasc Surg. 2014;60(4): 973-81 e971.

72. Gernigon M, Fouasson-Chailloux A, Colas-Ribas C, Noury-Desvaux B, Le Faucheur A, Abraham P. Test-retest reliability of GPS derived measurements in patients with claudication. Eur J Vasc Endovasc Surg. 2015;50(5):623-9.

73. Bertschi M, Celka P, Delgado-Gonzalo R, Lemay M, Calvo EM, Grossenbacher $\mathrm{O}$, Renevey $\mathrm{P}$. Accurate walking and running speed estimation using wrist inertial data. In: Annual IEEE International Conference on Engineering in Medicine and Biology Society (EMBC). 3rd ed; 2015. p. 8083-6.
74. Wilson AM, Lowe JC, Roskilly K, Hudson PE, Golabek KA, McNutt JW Locomotion dynamics of hunting in wild cheetahs. Nature. 2013;498(7453): 185-9.

75. Sangha O, Stucki G, Liang MH, Fossel AH, Katz JN. The self-administered comorbidity questionnaire: a new method to assess comorbidity for clinical and health services research. Arthritis Rheum. 2003;49(2):156-63.

76. Streibelt M, Schmidt C, Brünger M, Spyra K. Komorbidität im Patientenurteil - geht das? Validität eines Instruments zur Selbsteinschätzung der Komorbidität (SCQ-D). Orthopade. 2012;41:303-10.

77. Fillekes MP, Giannouli E, Kim EK, Zijlstra W, Weibel R. Towards a comprehensive set of GPS-based indicators reflecting the multidimensional nature of daily mobility for applications in health and aging research. Int J Health Geogr. 2019;18(1):17.

78. Bohannon RW, Tudini F. Unipedal balance test for older adults: a systematic review and meta-analysis of studies providing normative data. Physiotherapy. 2018;104(4):376-82.

79. Trampisch US, Franke J, Jedamzik N, Hinrichs T, Platen P. Optimal Jamar dynamometer handle position to assess maximal isometric hand grip strength in epidemiological studies. J Hand Surg [Am]. 2012;37(11):2368-73.

80. Lamb SE, Jorstad-Stein EC, Hauer K, Becker C. Development of a common outcome data set for fall injury prevention trials: the prevention of falls network Europe consensus. J Am Geriatr Soc. 2005:53(9):1618-22.

81. Lamprecht M, Fischer A, Stamm H. Sport Schweiz 2014 - Sportaktivität und Sportinteresse der Schweizer Bevölkerung. Magglingen: Bundesamt für Sport BASPO; 2014.

82. Altmeier D, Giannouli E. German translation and psychometric properties of the modified gait efficacy scale (mGES). Z Gerontol Geriatr. 2019; Epub ahead of print. https://doi.org/10.1007/s00391-019-01507-5.

83. Newell AM, VanSwearingen JM, Hile E, Brach JS. The modified gait efficacy scale: establishing the psychometric properties in older adults. Phys Ther. 2012;92(2):318-28.

84. Ustun TB, Chatterji S, Kostanjsek N, Rehm J, Kennedy C, Epping-Jordan J, Saxena S, von Korff M, Pull C, Project WNJ. Developing the World Health Organization disability assessment schedule 2.0. Bull World Health Organ. 2010;88(11):815-23.

85. McGraw KO, Wong SP. Forming inferences about some intraclass correlations coefficients. Psychol Methods. 1996;1(1):30-46.

86. Bland JM, Altman DG. Statistical methods for assessing agreement between two methods of clinical measurement. Lancet. 1986;1(8476):307-10.

87. Costa-Santos C, Antunes L, Souto A, Bernardes J. Assessment of disagreement: a new information-based approach. Ann Epidemiol. 2010; 20(7):555-61.

88. Kirkwood BR, Sterne JAC. Essential medical statistics. 2nd ed. Malden: Blackwell Science Ltd.; 2003.

89. Bonett DG. Sample size requirements for estimating intraclass correlations with desired precision. Stat Med. 2002;21(9):1331-5.

90. Piegorsch WW. Sample sizes for improved binomial confidence intervals Comput Stat Data Anal. 2004;46(2):309-16.

91. Anteil der Besitzer von Smartphones bzw. Tablets in der Schweiz in den Jahren von 2013 bis 2018. Y\&R/Statista [https://de.statista.com/statistik/ daten/studie/537944/umfrage/besitz-von-smartphone-bzw-tablet-in-derschweiz/].

92. US smartphone use in 2015. Pew Research Center [http://assets. pewresearch.org/wp-content/uploads/sites/14/2015/03/PI_Smartphones_04 01151.pdf].

93. Abelson JS, Symer M, Peters A, Charlson M, Yeo H. Mobile health apps and recovery after surgery: what are patients willing to do? Am J Surg. 2017; 214(4):616-22.

\section{Publisher's Note}

Springer Nature remains neutral with regard to jurisdictional claims in published maps and institutional affiliations. 